\title{
Evaluation of Route of Administration (Via Portal Vein) for Assessing the Role of Stem Cell Therapy in Cirrhosis of Liver
}

\section{Mamun Al Mahtab ${ }^{1}$, Ahmed Lutful Moben ${ }^{2}$, Md Abdur Rahim ${ }^{3}$, Md Ashraful Alam, Faiz Ahmad Khondaker", Sheikh Mohammad Noor-E-Alam ${ }^{1}$, Md Sakirul Islam Khan ${ }^{6}$, Md Zahurul Huq ${ }^{7}$, Musarrat Mahtab $^{8}$ and Sheikh Mohammad Fazle Akbar ${ }^{9,10 *}$}

${ }^{1}$ Department of Hepatology, Bangabandhu Sheikh Mujib Medical University, Dhaka, Bangladesh

${ }^{2}$ Department of Medicine, Kurmitola General Hospital, Dhaka, Bangladesh ${ }^{3}$ Department of Hepatology, Abdul Malek Ukil Medical College, Noakhali, Bangladesh ${ }^{4}$ Department of Hepatology, Shaheed Tajuddin Ahmed Medical College, Gazipur, Bangladesh

${ }^{5}$ Department of Hepatology, Shaheed Suhrawardy Medical College, Dhaka, Bangladesh

${ }^{6}$ Department of Anatomy and Embryology, Ehime University Graduate School of Medicine, Ehime, Japan

${ }^{7}$ Department of Anesthesiology, National Institute of Cardiovascular Diseases, Dhaka, Bangladesh

${ }^{8}$ Department of Biochemistry and Biotechnology, North South University, Dhaka, Bangladesh

${ }^{9}$ Department of Gastroenterology and Metabology, Ehime University Graduate School of Medicine, Ehime, Japan

${ }^{10}$ Researcher, Miyakawa Memorial Research Foundation, Tokyo, Japan

*Corresponding Author: Sheikh Mohammad Fazle Akbar, Department of Gastroenterology and Metabology, Ehime University Graduate School of Medicine, Ehime, Japan.
Received: September 14, 2020

Published: October 12, 2020

(C) All rights are reserved by Sheikh

Mohammad Fazle Akbar., et al.

\section{Abstract}

Aims and Objectives: The safety and efficacy of stem cell administration via portal vein was assessed in patients with cirrhosis of liver (LC)

Materials and Methods: In order to increase the numbers of white blood cell, 20 patients with liver cirrhosis were administered with granulocyte colony-stimulating factor at a dose of $60 \mathrm{IU}$, daily for 3 to 4 days. Stem cells were isolated from these patients as CD34-positive cells and variable numbers of autologous stem cells were injected to LC patients for once. Various hematological and clinical parameters of these patients were evaluated at point 0 (before administration of stem cells) and 1 and 3 months after administrations.

Results: None of these 20 patients died within 3 months of observation period. Regarding safety issues, there was no notable adverse event including acute liver failure related to stem cell therapy in these patients. Stem cell therapy had a dominant effect on ascites in this cohort. Although 7 of 20 patients had ascites at the start of therapy, 2 of these patients became ascites free at the end of 3 months of follow up. On the other hand, 5 patients of LC who did not have ascites at base line developed ascites during therapy. Different biochemical parameters of these patients remain mostly unchanged and did not show any significant difference dur to stem cell therapy. However, serum albumin level was increased significantly after 1 and 3 months.

Conclusion: The safety of stem cell therapy via portal vein has been shown here. The efficacy of stem cell therapy via portal vein may not be notable. However, observed effects of this therapy on ascites reduction in some patients and on effect on serum albumin indicate that repeated administration of stem cell therapy may be needed for maintaining quality of life.

Keywords: Cirrhosis of Liver (LC); Stem Cell Therapy; Hepatic Parenchyma 


\section{Introduction}

Cirrhosis of liver (LC) is characterized by alteration of hepatic parenchyma and distorted vascular supply represents an eminent public health problem. About one half of the chronic liver diseases related death can be attributed to LC. LC is regarded as an irreversible pathological process and medical management of LC is directed to symptomatic management $[1,2]$. Recent developments regarding cellular, immunological and molecular insights indicate that LC may not be completely irreversible and there remains to initiate innovative therapy against LC to minimize clinical anomaly related to LC $[3,4]$. Although anti-fibrotic drugs represent the best choice for treatment of LC, other approaches that may induce regeneration and immunological activation of hepatocytes may be considered. In line of this, investigators have used stem cell therapy, however, the outcome is yet to be validated by clinical trials of Phase I/II/III [5,6].

Bangladesh, the $7^{\text {th }}$ most populous country of the world, harbors about several hundred thousand patients with LC. Most of these patients die with severe down grading of life with ascites, frequent variceal bleeding, and hepatic encephalopathy; finally ending with liver failure or progressing to hepatocellular carcinoma (HCC). The economic burden of these patients is enormous [1,2].

To develop innovative therapy against LC, several sophisticated instruments and extremely safe Good Manufacturing Practices (GMP) levels facilities are required and these are yet to be available at Bangladesh for conducting proper cell therapy.

In this perspective, we planned a design of stem cell therapy that would ensure the proliferation or generation of stem cells by the action of growth factor endogenously in LC patients. This would be followed by isolation of stem cell in a closed chamber so that it remains free of contamination. These stem cells have been administered in hepatic artery in LC patients and the outcome was inspirable [7].

The liver receives blood supply from two major sources: hepatic artery and portal vein. As we accomplished stem cell therapy via hepatic artery, the present study was planned to assess the safety of efficacy of stem cell therapy via portal vein.

\section{Materials and Methods}

\section{Patients}

Stem cell therapy via portal vein was accomplished in 20 patients with LC. The diagnosis of LC was based on the diagnostic cri- teria provided by American Association for the Study of the Liver (AASLD), European Association for the Study of Liver (EASL) and Asia-Pacific Association for the Study of the Liver (APASL) [8-10]. LC was diagnosed based on biochemical parameters and final confirmation was accomplished by imaging of liver and/or liver biopsy. All of them were in a decompensated state and some of them had ascites and esophageal varices. None of them had HCC. Also, patients had no other concurrent diseases that may influence host immunity other than LC. Patients were not taking any immune modulators at the time clinical trial.

\section{Standard of care therapy for LC in Bangladesh}

In this study, all patients of LC received standard care therapy. The patients with LC those gave consent to receive this therapy were enrolled.

In Bangladesh the standard of care therapy was based on two main fundamentals; (1) treatment of etiology by antiviral drugs when infection with HBV or HCV can be confirmed and (2) symptomatic treatment of the patients: human albumin is given for substitution of low albumin; Bowel care is taken by lactulose. In the case of varices and variceal bleeding, all recommended therapies are employed based on the conditions of the patient.

Pretreatment administration of granulocyte colony stimulating factor (GCSF) to accentuate the frequencies of stem cell

All patients receive pretreatment granulocyte colony stimulating factor (GCSF) (60 IU) daily for 3 to 4 days around the umbilicus. The safety of the patients after administration of GCSF was properly evaluated and when there was no concern about GCSFinduced safety, the patients were provisionally selected for stem cell therapy via portal vein.

\section{Stem cell harvesting from patients}

Stem cell harvesting has been done after 3 to 4 daily administration of GCSF and this was affirmed by checking the counts of white blood cells. Stem cell was isolated based on a close chamber-related isolation method, exactly as described by the manufacturer and optimized by us. The apheresis machine (COM.TEC, Fresenius Kabi AG, Hamburg, Germany) was used to get the enriched population of stem cells and this minimized the possibility of contamination. A commercial kit called P1YA kit (Fresenius Kabi AG, Hamburg, Germany) has been used for harvesting stem cells from peripheral blood. After counting and confirming the viability of stem cells, these were administered to patients. 
The methodology of stem cell therapy via blood vessel has been described in our previous communication. In this study, the stem cells were administered into portal vein in spite of hepatic artery. A catheter was inserted into the portal vein. Acid citrate dextrose (ACD)-1 (Fresenius Kabi AG) has usually been used as an anticoagulant during a procedure at a ratio of 12-14:1. Injection with calcium gluconate $(10 \%)$ was given to counter the adverse reaction of ACD-1. The patient was under continuous monitoring by cardiac monitor throughout the procedure. The machine is a continuous type of apheretic machine where blood is collected and undergo centrifugation for the collection of stem cells. At the same time, other types of blood cells and plasma are returned to the circulation of the patient. Approximately 60 to $65 \mathrm{~mL}$ stem cells have been collected and the numbers of stem cells were calculated by flow cytometry (Bacton Dickenson FACSVerse, Bacton Dickenson Biosciences, San Jose, CA, USA).

\section{Follow-up of the patients}

The initial follow-up was planned for after 3 months and then secondary follow up will be accomplished after 6 and 12 months. During the initial follow up of 3 months, general features of the patients were analyzed by frequent hospital visits. Also, the parameters of liver and kidney functions were assessed periodically and data of different parameters before the cell therapy and after 1 and 3 months were collected for analysis. Careful consideration was provided about the extent of ascites.

\section{Statistical analysis}

Statistics were performed using the average values, and all data are reported as means \pm SD (except mentioned elsewhere). The data on serum albumin level was statistically analyzed using the one-way repeated measurement of analysis of variance (ANOVA), and the Tukey-Kramer post-hoc comparisons. Data serum albumin levels are expressed as means \pm SEM, and statistical significance was set at $\mathrm{p}<0.05$. The number of patients in each observation point is described in the table 1.

\section{Results}

The mean age of the patient was 51 years (standard deviation, 11.5 years). There were 15 males and 5 females. The possible causes of LC were hepatitis B virus in 6 patients, hepatitis C virus in
1 patient, non-alcoholic steatohepatitis in 2 patients, cryptogenic in others.

Granulocyte colony-stimulating factor in positively regulating blood cells

The GCSF induced increased numbers of WBC in all patients, although the effects of GCSF were highly variable among patients [7]. The mean value of WBC was about 10074 cells/ $\mu$ l (range: 2200 to 13,000 cells $/ \mu \mathrm{l}$ ) before the start of administration of GCSF. After end of GCSF administration, the mean value of WBC raised to 26,000 cells $/ \mu \mathrm{l}$ (4000 - 22500 cells/ $\mu \mathrm{l}$ ). The etiological agents and patients' clinical conditions had no convincible role on GCSFinduce cell modulation. Usually, 6 to 8 injections with GCSF were enough to have doubling or tripling of WBC counts. Due to stimulation with GM-CSF, the levels of WBC increased to about 3 folds (mean value; 33000 cells $/ \mu \mathrm{l}$ ). The number of stem cells isolated by close chamber procedure also varied considerably among patients. However, as a standard practice, we used at least 100,000 stem cells per patient.

\section{Survival outcome}

The administration of stem cell via portal vein was safe and no adverse effects have been recorded in any patient, as that has been mentioned in other studies $[12,13]$. A total of 20 patients were enrolled in this study and there was no death in any of these patients within the study period.

\section{Effect of stem cell therapy on biochemical parameters}

The biochemical data of the patients have been summarized in table 1 . We could not document any significant change in the levels of serum bilirubin, SGPT, INR, and serum creatinine levels before and at 1 and 3 months after therapy (Table 1 ). The only notable change by stem cell therapy was found in serum albumin level. The levels of albumin increased one month after therapy compared to basal levels of serum albumin and remained slight higher at 3 months after commencement of therapy.

\section{Ascites and survival}

Regarding ascites, 7 patients with LC had ascites when they were enrolled. Out of these 7 patients ascites became invisible in 5 patients, whereas, 2 patients those did not have ascites before start of therapy developed ascites. 
Evaluation of Route of Administration (Via Portal Vein) for Assessing the Role of Stem Cell Therapy in Cirrhosis of Liver

\begin{tabular}{|l|c|c|c|c|c|c|}
\hline & Bilirubin & SGPT & Albumin & INR & PT & Creatinine \\
\hline Basal & $2.2 \pm 2.5$ & $38 \pm 25$ & $2.63 \pm 0.45$ & $1.67 \pm 0.53$ & $20.24 \pm 6.63$ & $1.09 \pm 0.35$ \\
\hline One month after start of therapy & $1.81 \pm 1.55$ & $35.55 \pm 14.3$ & $3.03 \pm 0.48^{*}$ & $2.0 \pm 2.2$ & $18.41 \pm 6.0$ & $1.03 \pm 0.36$ \\
\hline Three months after start of therapy & $2.03 \pm 1.80$ & $33.0 \pm 12.8$ & $3.12 \pm 0.39^{*}$ & $2.02 \pm 2.39$ & $17.58 \pm 6.0$ & $1.04 \pm 0.37$ \\
\hline
\end{tabular}

Table 1: Biochemical parameters of patients with liver cirrhosis at different time points.

SGPT: Serum Glutamic-Pyruvic Transaminase; INR: International Normalized Ratio; PT: Prothrombin Time. p $<0.001$.

\section{Discussion}

This is our $3^{\text {rd }}$ approach to develop an evolving and novel therapeutic approach for LC [7,11]. During last 5 years, we have activated immunocytes in vivo or used stem cell to have therapeutic effect in LC patients. The present study used a new mode of administration of stem cells for treatment of LC. The role of stem cell as regenerative potentials is not yet clarified as we have neither checked production of new stem cells in situ nor assessed the migration of injectable stem cells to liver. Thus, we are unable to specify the real implication of stem cells in LC. However, this provided an impression that cells prepared in close chamber is a safe endeavor to induce cells therapy in developing and resource-constrained countries. The safety issue would initiate several novel therapeutic approaches in these countries those harbor millions of patients with intractable diseases, but lack sophisticated human GMP level facilities.

In our previous article in which we administrated stem cells via hepatic artery induced positive impact regarding serum albumin and ascites. In this study, the positive impact on serum albumin levels were visible. It might indicate that multiple settings of therapy via portal vein would be necessary. The loss of ascites in some patients and getting ascites in others may indicate a complex role of stem cell therapy in LC patients [14-17].

There may be multiple logics of the positive impact of cell therapy in LC patients. In one hand, investigators have mentioned that stem cell may be converted to healthy hepatocytes in the diseased liver. On the contrary, the immune modulatory effects of stem cell in LC liver may be beneficial to LC patients. However, this study was not designed to assess the conversion of infused stem cells as hepatocytes in the liver of LC patients due to the concern of safety and technical factors. The next, the stem cells were harvested in a closed chamber so that these may not be contaminated as good manufacturing practice (GMP) is yet to be introduced for cell therapy in Bangladesh.

\section{Conclusion}

Stem cell therapy is at its infancy and more works are needed to optimize this new and novel therapy for LC. Usage of different population of stem cell and alteration of protocol may resolve some of the problems. In addition, the entity and concept of stem cell therapy for LC as regenerative therapy in under challenge. More basic and fundamental studies would be warranted to optimize about this.

\section{Source of Support}

The study was partially supported by a grant from Bangabandhu Sheikh Mujib Medical University, Dhaka, Bangladesh to Mamun Al Mahtab.

\section{Conflict of Interest}

None.

\section{Bibliography}

1. Barnett R. "Liver cirrhosis". Lancet 392.10144 (2018): 275.

2. Romanelli RG and Stasi C. "Recent Advancements in Diagnosis and Therapy of Liver Cirrhosis". Current Cancer Drug Targets 17.15 (2016): 1804-1817.

3. Sun M and Kisseleva T. "Reversibility of liver fibrosis". Clinics and Research in Hepatology and Gastroenterology 39.1.01 (2015): S60-S63.

4. Jung YK and Yim HJ. "Reversal of liver cirrhosis: current evidence and expectations". The Korean Journal of Internal Medicine 32.2 (2017): 213-228.

5. Newsome PN., et al. "Granulocyte colony-stimulating factor and autologous CD133-positive stem-cell therapy in liver cirrhosis (REALISTIC): an open-label, randomised, controlled phase 2 trial". The Lancet Gastroenterology and Hepatology 3.1 (2018): 25-36.

6. Lin PC., et al. "A proposed novel stem cell therapy protocol for liver cirrhosis". Cell Transplantation 24.3 (2015): 533-540. 
7. Al Mahtab M., et al. "Stem Cell Therapy for Cirrhosis of Liver in Bangladesh: Specific Design Compatible for Developing Country". Euroasian Journal of Hepato-Gastroenterology 8.2 (2018): 121-125.

8. Terrault NA., et al. "American Association for the Study of Liver Diseases. AASLD guidelines for treatment of chronic hepatitis B". Hepatology 63.1 (2016): 261-283.

9. European Association for Study of Liver. "EASL Clinical Practice Guidelines: management of hepatitis C virus infection". The Journal of Hepatology 60.2 (2014): 392-420.

10. Sarin SK., et al. "Asian-Pacific clinical practice guidelines on the management of hepatitis B: A 2015 update". Hepatology International 10.1 (2016): 1-98.

11. Al Mahtab M., et al. "Therapy Targeting Stem Cell in Patients with Decompensated Cirrhosis of Liver in a Tertiary Treatment Care Center of Bangladesh". Euroasian Journal of HepatoGastroenterology 7.1 (2017): 113-115.

12. Kaibori M., et al. "Stimulation of liver regeneration after hepatectomy in mice by injection of bone marrow mesenchymal stem cells via the portal vein". Transplantation Proceedings 44.4 (2012): 1107-1109.

13. Huang XL., et al. "Clinical outcome of autologous hematopoietic stem cell infusion via hepatic artery and portal vein in patients with end-stage liver disease". Chinese Medical Sciences Journal 29.1 (2014): 15-22.

14. Nicolas C., et al. "Stem Cell Therapies for Treatment of Liver Disease". Biomedicines 4.1 (2016): 2.

15. Saha BK., et al. "Therapeutic implications of granulocyte colony stimulating factor in patients with acute-on-chronic liver failure: increased survival and containment of liver damage". Hepatology International 11.6 (2017): 540-546.

16. Pai M., et al. "Autologous infusion of expanded mobilized adult bone marrow-derived CD34+ cells into patients with alcoholic liver cirrhosis". The American Journal of Gastroenterology 103.8 (2008): 1952-1958.

17. Sellamuthu S., et al. "In vitro transdifferentiation of human umbilical cord derived hematopoietic stem cells into hepatocyte like cells using combination of growth factors for cell-based therapy". Cytotechnology 63 (2011): 259-268.

\section{Assets from publication with us}

- Prompt Acknowledgement after receiving the article

- Thorough Double blinded peer review

- Rapid Publication

- Issue of Publication Certificate

- High visibility of your Published work

Website: www.actascientific.com/

Submit Article: www.actascientific.com/submission.php Email us: editor@actascientific.com

Contact us: +919182824667 